\title{
Dual-band asymmetric planar dipole antenna backed by conducting reflector for WLAN applications
}

\author{
Suguru Kojima, Takuji Arima, and Toru Uno ${ }^{\text {a) }}$ \\ Department of Electrical and Electronic Engineering, Tokyo University of \\ Agriculture and Technology, Tokyo 184-8588, Japan \\ a)uno@cc.tuat.ac.jp
}

Abstract: This paper proposes a dual-band planar antenna composed of asymmetric dipole elements backed by a conducting reflector that is realized by incorporating a LC trap circuit. The proposed antenna achieves the $2.4 \mathrm{GHz}$ and $5 \mathrm{GHz}$ dual-band operations for the purpose of WLAN applications. It is shown that the measured gains were $3.0 \mathrm{dBi}$ at $2.44 \mathrm{GHz}$, $5.3 \mathrm{dBi}$ at $5.25 \mathrm{GHz}$, and $5.8 \mathrm{dBi}$ at $5.6 \mathrm{GHz}$, respectively. It is also confirmed that this antenna approximately retains a unidirectional property and a superdirectivity as well.

Keywords: superdirective, low-profile, dual-band, LC trap circuit, IoT, WLAN

Classification: Antennas and Propagation

\section{References}

[1] S. Kojima, T. Arima, and T. Uno, "Low-profile supergain antenna composed of asymmetric dipole elements backed by planar reflector for IoT applications," IEICE Trans. Commun., vol. E102-B, no. 4, pp. 884-890, Apr. 2019. DOI:10. 1587/transcom.2018EBP3112

[2] S. Kojima, T. Arima, and T. Uno, "Improvement of bandwidth characteristics of low-profile superdirective antenna composed of asymmetric dipole element backed by planar reflector," IEICE Commun. Express, vol. 8, no. 12, pp. 458461, July 2019. DOI:10.1587/comex.2019GCL0005

[3] T. Huynh and K.-F. Lee, "Single-layer single-patch wideband microstrip antenna," Electron. Lett., vol. 31, no. 16, pp. 1310-1312, 1995. DOI:10.1049/ el:19950950

[4] K.-F. Lee, S. L. S. Yang, and A. A. Kishk, "Dual- and multiband U-slot patch antennas," IEEE Antennas Wireless Propag. Lett., vol. 7, pp. 645-647, 2008. DOI:10.1109/LAWP.2008.2010342

[5] P. Salonen, M. Keskilammi, and M. Kivikoski, "Single-feed dual-band planar inverted-f antenna with u-shaped slot," IEEE Trans. Antennas Propag., vol. 48, no. 8, pp. 1262-1264, Aug. 2000. DOI:10.1109/8.884498

[6] D. Smith, "The trap-loaded cylindrical antenna," IEEE Trans. Antennas Propag., vol. 23, no. 1, pp. 20-27, Jan. 1975. DOI:10.1109/TAP.1975.1141000

[7] http://www.engineering-eye.com/MAGNA_TDM/ (MAGNA/TDM Ver. 8.3, ITOCHU Techno-Solutions Corporation).

[8] S. R. Best, E. E. Altshuler, A. D. Yaghjian, J. M. McGinthy, and T. H. 
O’Donnell, “An impedance-matched 2-element superdirective array," IEEE

Antennas Wireless Propag. Lett., vol. 7, pp. 302-305, 2008. DOI:10.1109/

LAWP.2008.921372

\section{Introduction}

The antenna for the IoT is required to be a low-profile for the benefit of high applicability to the things. The unidirectional radiation property is also required to reduce the influence of the surrounding objects such as a human body and a conducting plate. For this end, the authors have proposed a planar low-profile antenna composed of an asymmetric dipole element backed by a conducting reflector [1]. This antenna exhibits both the superdirectivity and unidirectional property, however, the operating bandwidth was relatively narrow. In [2], the bandwidth has been enhanced by incorporating a notch structure implemented in the ground plane element and a T-type monopole element. It has been shown this antenna retains the unidirectional property and also superdirectivity. The purpose of this paper is to add a dual-band performance to the original antenna for WLAN application.

A microstrip patch antenna [3, 4] and a planar inverted-F antenna [5] has been investigated and proposed as the dual-band low-profile antennas. However, the structures of the feeding parts are unsuitable for IoT applications in comparison with the original antenna proposed by the authors in which the feed element lies on the same plane with the radiation elements. In this paper, the dual-band performance is realized by adding the LC trap circuits to the primary element and ground plane elements in the original antenna [6] in order to reduce the inherent high applicability to the IoT and its appearance and mobility as well. It is shown that the developed antenna exhibits dual-band characteristics and maintains the unidirectional property. It is also confirmed that the superdirective property is approximately retained. All of the numerical simulations were performed using a commercially provided software based on the Finite-Difference Time-Domain (FDTD) method [7].

\section{Proposed antenna and its characteristics}

Fig. 1 shows the geometry of the proposed antenna and a photo of an experimental antenna where the trap circuit and the feeding point are enlarged. The antenna is composed of asymmetric dipole elements which fundamentally consist of an L6 $\times$ W2 monopole element which is divided into two sub-parts, an L4 $\times$ W1 ground plane element which is divided into three sub-parts, and a planar reflector. The LC lumped circuit (indicated by 'Trap' in the figure) is equipped at the $2 \mathrm{~mm}$ slits between three sub-parts in the ground plane element, and in the monopole element as well. All of the antenna elements are placed on the $1 \mathrm{~mm}$ thickness dielectric substrate, and are backed by the same width and slightly long reflector at the distance D. The monopole element is fed at the center of the ground plane, and the feeding gap was set to $1 \mathrm{~mm}$ for both experiment and calculation. 
These elements were made by a copper foil whose conductivity is approximately $5.8 \times 10^{7} \mathrm{~S} / \mathrm{m}$, however in numerical calculations, they are assumed as a PEC (Perfect Electric Conductor) for simplicity. The relative permittivity and the loss tangent $(\tan \delta)$ of the substrate are measured at $2.4 \mathrm{GHz}$ and obtained as 4.3 and 0.018 respectively. In the numerical calculations the corresponding conductivity are assumed to be constant all over the target frequency range, that is $2.4 \mathrm{GHz}$ and $5 \mathrm{GHz}$ bands.

Since the resonance frequency and impedance matching are fundamentally determined by the total length of the ground plane element and the monopole antenna element, that is L4 + L6 [1], this paper investigates to get the dual band property by controlling the current on these elements, specifically realizing the longer length for lower frequency band and shorter length for higher frequency band. For this purpose, the authors used the trap circuit composed of an inductor and two capacitors which are realized by a LQW-type inductor provided by Murata Manufacturing Co. Ltd. and a chip capacitor provided by NGK Electronics Devices Inc. respectively and are connected at some appropriate positions as shown in Fig. 1. These constants of the element were determined in a way that the circuit operates as short at $2.4 \mathrm{GHz}$ band and as open at $5 \mathrm{GHz}$ band as a whole structure including antenna elements. The resultant values are $\mathrm{L}=4.7 \mathrm{nH}, \mathrm{C} 1=0.15 \mathrm{pF}$, $\mathrm{C} 2=0.75 \mathrm{pF}$. Then, although the currents on L2-part and L4-part in Fig. 1 resonate near $5 \mathrm{GHz}$ and $2.4 \mathrm{GHz}$, respectively, the radiation pattern at $5 \mathrm{GHz}$ band does not indicate unidirectional property because the current at $5 \mathrm{GHz}$ band which is approximately a second harmonic component of $2.4 \mathrm{GHz}$, considerably flows in (L4-L2)-part. Therefore (L4-L2)-part was divided into two parts again and inserted the same trap circuit in this paper.

The measured and calculated VSWRs are shown in Fig. 2. The parameters of the antenna were set as $\mathrm{D}=3 \mathrm{~mm}, \mathrm{~L} 1=62 \mathrm{~mm}, \mathrm{~L} 2=18 \mathrm{~mm}, \mathrm{~L} 3=29 \mathrm{~mm}$, $\mathrm{L} 4=40 \mathrm{~mm}, \mathrm{~L} 5=8 \mathrm{~mm}, \mathrm{~L} 6=13 \mathrm{~mm}, \mathrm{~L} 7=17 \mathrm{~mm}, \mathrm{~W} 1=25 \mathrm{~mm}, \mathrm{~W} 2=1 \mathrm{~mm}$. It is found that the measured result agrees fairly well with the calculated result for both frequency bands, and that the VSWR is less than 2.5. It is also found that the bandwidth at $2.4 \mathrm{GHz}$ band is relatively narrow but is widened at $5 \mathrm{GHz}$ band. Its physical reason is difficult to describe definitely, but will mainly be resulted from the trap circuit, that is, the antenna operates through the narrowband short circuits at $2.4 \mathrm{GHz}$ band and the open circuits at $5 \mathrm{GHz}$ band which are connected between two separate conductors.

Fig. 3 shows the radiation patterns at $2.44 \mathrm{GHz}, 5.25 \mathrm{GHz}$, and $5.6 \mathrm{GHz}$ in $\mathrm{x}-\mathrm{z}$ plane and $y-z$ plane, respectively. In is found that the unidirectional property is reasonably achieved for all frequencies. The measured gains were $3.0 \mathrm{dBi}$ at $2.44 \mathrm{GHz}, 5.3 \mathrm{dBi}$ at $5.25 \mathrm{GHz}$ and $5.8 \mathrm{dBi}$ at $5.6 \mathrm{GHz}$. It is not shown here, but the phases of the currents on the antenna element and the reflector are different from about 180 degrees in phase. Thus, both the radiation antenna and the reflector compose 2-element superdirective array [8] as described in [1], considering together with the gain and the radiation pattern. 


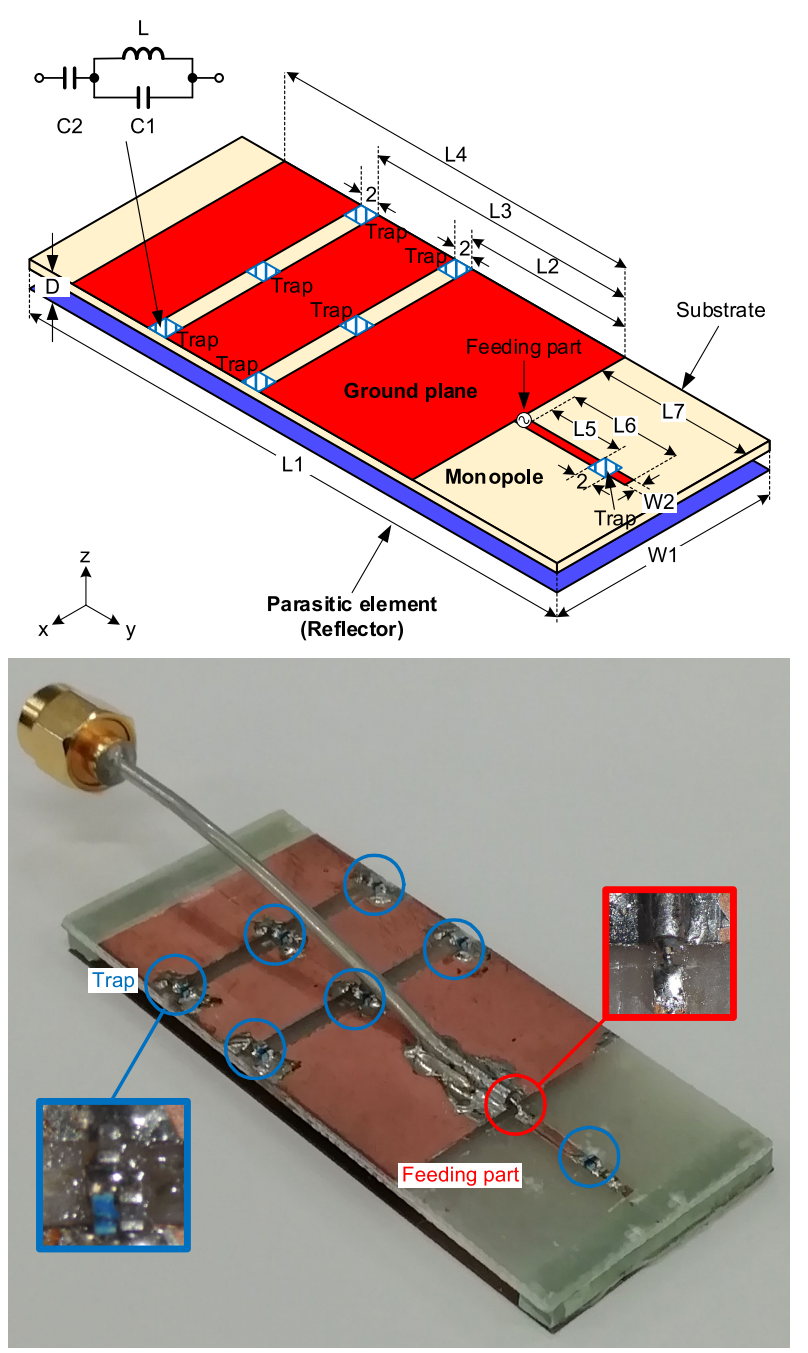

Fig. 1. Geometry of the proposed antenna.

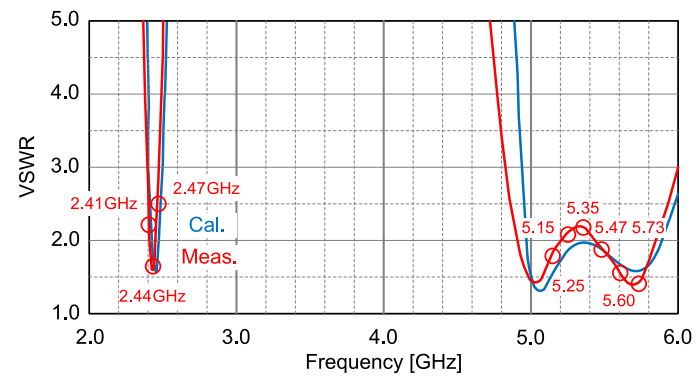

Fig. 2. VSWR.

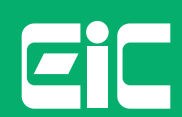

๑ IEICE 2020

DOI: 10.1587/comex.2019XBL0153

Received December 3, 2019

Accepted December 16, 2019

Publicized December 27, 2019

Copyedited March 1, 2020 

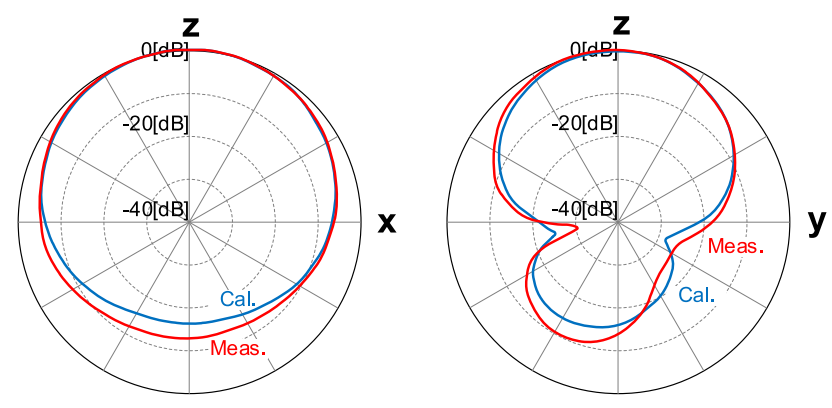

(a)
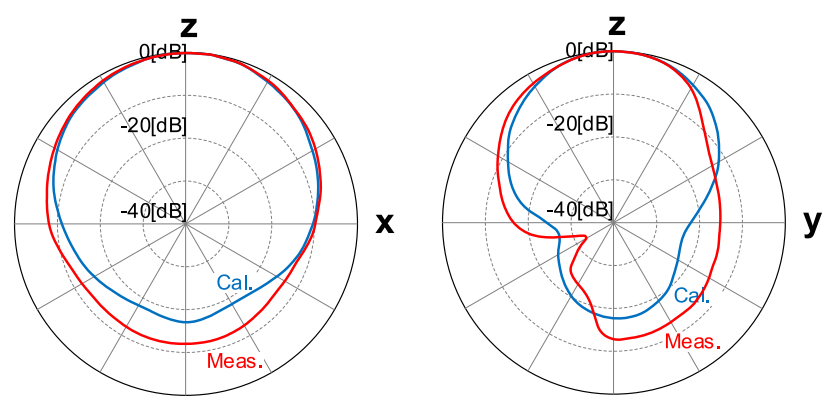

(b)
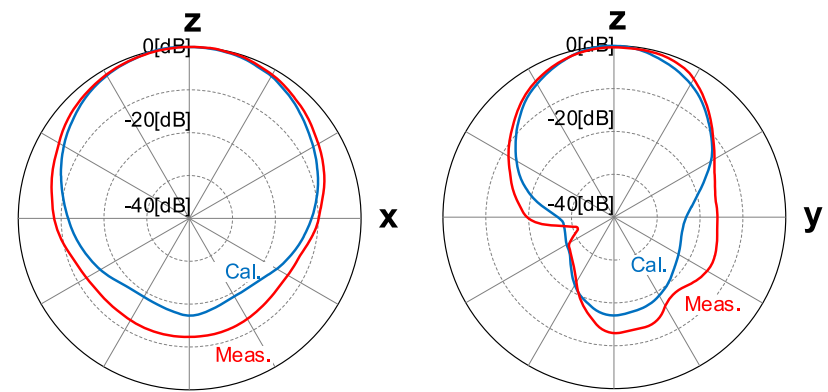

(c)

Fig. 3. Radiation patterns. (a) Radiation patterns at $2.44 \mathrm{GHz}$. (b) Radiation patterns at $5.25 \mathrm{GHz}$. (c) Radiation patterns at $5.6 \mathrm{GHz}$.

\section{Conclusion}

This paper has proposed the $2.4 \mathrm{GHz}$ and the $5 \mathrm{GHz}$ dual-band low-profile antenna for WLAN applications. The proposed antenna is achieved unidirectional radiation pattern and dual-band by utilizing trap circuits on the antenna. It has also been shown that the superdirectiveity is approximately retained. Thus, it is expected that the proposed antenna may be successfully applied to IoT applications. 